British Journal of Psychiatry (1992), 161, 859-868

\title{
Correspondence
}

Editor: Ian Pullen

Contents: Periodic psychosis associated with menstrual cycle/Syndromes of schizophrenia on factor analysis/Propofol and ECT/Clozapine/Multiple personality disorder/The neglect of anger in Western psychiatry/The Northfield Experiments/Self-neglect in adult life/The designation of black people in psychiatric research/Episodic dyscontrol/Applying Roberts' framework/Botulinium toxin in a case of severe tardive dyskinesia mixed with dystonia

\section{Periodic psychosis associated with menstrual cycle}

SIR: All monthly cycles are not menstrual. It has long been established that regularly recurring episodes of depression, catatonic schizophrenia, epileptic fits, bleeding, pyrexia, etc., with monthly periodicity can occur in men (Reimann, 1963; Richter, 1965). In women, when the dates of onset of menstruation and of psychological disturbance are accurately recorded over a year or more, two distinct rhythms can be discerned, which may gradually draw apart. In both sexes, syndrome rhythms of other than 30 days $(6$, 42,70 , etc.) may be seen, and these are unaffected by the menopause. Furthermore, the menstrual cycle is metabolically (endocrinologically) divisible into at least two or three sections: follicular, luteal, and menstruating, which are different, so that the same psychological phenomena are unlikely to be associated with all phases of the cycle. In other words, it is important in probing the mechanism of illness and treating it effectively to distinguish between disturbance precisely associated with one phase of the menstrual cycle only, and disturbances which recur independently but with about the same wavelength.

Lovestone (Journal, September 1992, 161, 402404) claims his patient's psychosis was associated with the menstrual cycle, but his chart of seven periods and six mental episodes does not show this convincingly: three periods fell in the middle of an episode, one occurred in the middle of a remission, and two began as the episode began. In the absence of any other evidence his claim is inaccurate and fogs the pathology. We expect more accurate timing, some hormonal measures perhaps, but particularly a demonstration that blocking the menstrual cycle interferes with the mental episode. If an acute hormonal intervention during a natural remission will provoke mental symptoms briefly, so much the better for clarifying the illness.

Others (e.g. Endo et al, 1978) make Dr Lovestone's mistake, whereas Gerada \& Reveley (1988) report a case with precise, always premenstrual, timing and brief spontaneous remission in an amenorrhoeic cycle, together with 'cure' by dydrogesterone. My case (Crammer, 1986) similarly had an episode of depressive type filling 14 days preceding the onset of each period, except in one spontaneous amenorrhoeic cycle, and was 'cured' by stopping the cycle with an oestradiol implant. However, the illness appeared to be related to cortisol level rather than to sex hormone disturbance. There is something rather complex to be disentangled here. It is relevant to the pathophysiology of premenstrual states. We shall only make progress by recognising that monthly cyclical mental states can arise through more than one physical disturbance, some not linked to the sex cycle at all, others linked, but by some indirect hormonal pathology, and so on.

Crammer, J. L. (1986) Premenstrual depression, cortisol and oestradiol treatment. Psychological Medicine, 16, 451-455.

Endo, M., Daiguj, M., Asano, Y., et al (1978) Periodic psychosis recurring in association with the menstrual cycle. Journal of Clinical Psychiatry, 39, 456-466.

Gerada, C. \& Reveley, A. (1988) Schizophreniform psychosis associated with the menstrual cycle. British Journal of Psychiatry, 152, 700-702.

Reimann, H. A. (1963) Periodic Diseases. Philadelphia: F. A. Davis. Richter, C. P. (1965) Biological Clocks in Medicine and Psychiatry. Springfield: C.C. Thomas.

South Grange

Steeple Aston

Bicester OX6 3SS

SIR: I read with interest Lovestone's article (Journal, September 1992, 161, 402-404), and would like to comment on the association of the recurrent psychosis in the premenstrual period with an increased blink rate. 
Elevated blood 5-HT levels have been reported in psychosis in a number of studies (Garelis et al, 1975; DeLisi et al, 1981; Stahl et al, 1983). Blood 5-HT levels tend to increase in the premenstrual period in normal women (Rapkin et al, 1987). Increased 5-HT levels in the premenstrual period may therefore contribute to the relapsing of the psychosis, especially the negative symptoms.

There is also evidence for the serotoninergic modulation of blinking in animal experiments (Dursun \& Handley, 1991) The 5-HT $/ 5-\mathrm{HT}_{1 \mathrm{C}}$ receptor antagonist ritanserin abolished blinking induced by intracerebroventricular injection of thryotropinreleasing hormone, whereas haloperidol did not (Dursun \& Handley, 1991). This experimental data also supports Dr Lovestone's observation about the inactivity of adequate dosage of dopamine antagonists to antagonise blinking.

DeLisi, L. E., Neckers, L. M., Weinberg, D. R., et al (1981) Increased whole blood serotonin concentrations in chronic schizophrenic patients. Archives of General Psychiatry, 38, 647-650.

Dursun, S. M. \& Handley, S. L. (1991) Tic-like behaviours induced by TRH-amide: differential effects of ritanserin and haloperidol. British Journal of Pharmacology, 102, 229P.

Garelis, E., Gillin, J., WyatT, R. J., et al (1975) Elevated blood serotonin concentrations in unmedicated chronic schizophrenic patients: a preliminary study. American Journal of Psychiatry 132, 184-186.

Rapkin, A. J., Edelmuth, E., Chang, L. C., el al (1987) Wholeblood serotonin in premenstrual syndrome. Obstetrics and Gynaecology, 70, 533-537.

Stahl, S. M., Woo, D. J., Mefrord, I. N., et al (1983) Hyperserotonemia and platelet serotonin uptake and release in schizophrenia and affective disorders. American Journal of Psychiatry, 140, 26-30.

Pharmaceutical Sciences Institute

SERDAR M. DURSUN

Aston University, Aston Triangle

Birmingham B4 7 ET

\section{Syndromes of schizophrenia on factor analysis}

SIR: Several studies in recent years have investigated the grouping of schizophrenic symptoms by factor and cluster analyses (Liddle, 1987; Mortimer et al, 1990; Liddle \& Barnes, 1990; Peralta et al, 1992). The use of these techniques is a welcome trend. Published studies reveal two main applications: (a) elucidation of the factorial structure of clinical rating scales in which all the items are analysed; and (b) determination of symptom clusters in schizophrenia where the choice of items is determined by investigators. However, there are several limitations to the use of factor analysis for interpretation of findings and classifying syndromes, some of which we wish to highlight.
Firstly, the choice of sample is important. Schizophrenic in-patients differ from matched controls in the community on several parameters, especially the types and severity of symptoms and indices of deterioration (Soni et al, 1992). Factor analysis on such patients will produce results biased towards chronic residual psychopathology. If the inpatient sample is then further selected on the basis of severity of symptoms, only the most deteriorated patients would be included in the data analysis. Symptoms seen during acute exacerbation of schizophrenic illness often differ from those seen during remission; to our knowledge this has not been systematically investigated. Besides, symptoms during the earlier years of illness differ from those seen in older schizophrenics, presumably due to changes related to ageing and other factors. All these factors could affect the outcome of factor analysis.

Secondly, the choice of variables must be considered carefully. The initial correlation matrix and reliability analysis are useful tools for choosing variables on the basis of simplicity and parsimony. It is interesting that most factor analyses have been unable to elicit an affective syndrome, although clinical experience in the community suggests that schizophrenic patients with clinically significant depression or anxiety are far from rare. The choice of clinical rating scales is equally important and a recent study (Peralta et al, 1992) reported three syndromes of acute schizophrenia using SANS and SAPS, both of which exclude affective items.

Thirdly, factor analysis, like several other multivariate statistical procedures, requires rigid control of distribution characteristics of variables. Uncritical acceptance of minimum eigenvalues for the number of factors to be resolved (which in most statistical packages is one by default) may confound the factor structure and composition. It must be remembered that factor analysis tries to group variables into meaningful factors which reflect certain underlying constructs. The different factors should in no way be construed as being independent syndromes unless supported by associated data; all that can be said about the factors is that certain symptoms 'run together' in clinical practice. This is clearly indicated by a scrutiny of the factor coefficients, which invariably reveal considerable overlap.

It is clear that great caution is necessary in using factor analysis and in interpreting its results in clinical practice. Researchers should document and report the various characteristics of samples so that their results can be compared with other reports. It should not be assumed that syndromes elicited by factor analysis are necessarily independent; in most cases there is considerable overlap. Finally, it is 